\title{
EXAMINING THE ROLE OF ENVIRONMENT IN A COMPREHENSIVE SAMPLE OF COMPACT GROUPS
}

\author{
Lisa May Walker ${ }^{1}$, Kelsey E. Johnson ${ }^{1}$, Sarah C. Gallagher ${ }^{2}$, Jane C. Charlton ${ }^{3}$, \\ ANN E. HORNSCHEMEIER ${ }^{4}$, AND JOHN E. HibBARD ${ }^{5}$ \\ ${ }^{1}$ Department of Astronomy, University of Virginia, Charlottesville, VA 22904, USA \\ ${ }^{2}$ Department of Physics and Astronomy, University of Western Ontario, London, ON N6A 3K7, Canada \\ ${ }^{3}$ Department of Astronomy and Astrophysics, Pennsylvania State University, University Park, PA 16802, USA \\ ${ }^{4}$ Laboratory for X-Ray Astrophysics, NASA Goddard Space Flight Center, Greenbelt, MD 20771, USA \\ ${ }^{5}$ National Radio Astronomy Observatory, Charlottesville, VA 22903, USA \\ Received 2011 October 11; accepted 2012 January 5; published 2012 February 9
}

\begin{abstract}
Compact groups, with their high number densities, small velocity dispersions, and an interstellar medium that has not been fully processed, provide a local analog to conditions of galaxy interactions in the earlier universe. The frequent and prolonged gravitational encounters that occur in compact groups affect the evolution of the constituent galaxies in a myriad of ways, for example, gas processing and star formation. Recently, a statistically significant "gap" has been discovered in the mid-infrared (MIR: 3.6-8 $\mu \mathrm{m}$ ) IRAC color space of compact group galaxies. This gap is not seen in field samples and is a new example of how the compact group environment may affect the evolution of member galaxies. In order to investigate the origin and nature of this gap, we have compiled a larger sample of 37 compact groups in addition to the original 12 groups studied by Johnson et al. (yielding 174 individual galaxies with reliable MIR photometry). We find that a statistically significant deficit of galaxies in this gap region of IRAC color space is persistent in the full sample, lending support to the hypothesis that the compact group environment inhibits moderate specific star formation rates. Using this expanded sample, we have more fully characterized the distribution of galaxies in this color space and quantified the low-density region more fully with respect to MIR bluer and MIR redder colors. We note a curvature in the color-space distribution, which is fully consistent with increasing dust temperature as the activity in a galaxy increases. This full sample of 49 compact groups allows us to subdivide the data according to physical properties of the groups. An analysis of these subsamples indicates that neither projected physical diameter nor density shows a trend in color space within the values represented by this sample. We hypothesize that the apparent lack of a trend is due to the relatively small range of properties in this sample, whose groups have already been pre-selected to be compact and dense. Thus, the relative influence of stochastic effects (such as the particular distribution and amount of star formation in individual galaxies) becomes dominant. We analyze spectral energy distributions of member galaxies as a function of their location in color space and find that galaxies in different regions of MIR color space contain dust with varying temperatures and/or polycyclic aromatic hydrocarbon emission.
\end{abstract}

Key words: galaxies: clusters: general - galaxies: evolution - galaxies: interactions - galaxies: statistics - infrared: galaxies

Online-only material: color figures

\section{INTRODUCTION}

The majority of galaxies reside in poor groups of galaxies (Mulchaey 2000). With number densities similar to those seen in the centers of rich clusters, compact groups constitute a high-density sub-category of poor groups (Hickson 1982). This environment is thought to provide a local analog to conditions in the early universe when the average galaxy density was higher and interactions were more common and prolonged.

Despite their similar densities, compact groups exhibit marked differences from the centers of clusters. This is especially true with regard to the interstellar medium (ISM), a crucial component in the process of galaxy evolution. For instance, the centers of clusters tend to have no neutral gas, while compact groups typically contain at least some neutral gas, though they are known to be $\mathrm{H}$ I deficient as a class (Verdes-Montenegro et al. 2001). These H I deficiencies in compact groups are likely related to the ISM behavior in the outskirts of clusters, where the degree of $\mathrm{H} \mathrm{I}$ deficiency is correlated with the radial distance of the galaxy to the cluster's center (Giovanelli \& Haynes 1985). In addition, both compact groups and clusters show a correlation between $\mathrm{H}$ I deficiency and X-ray detectability (Giovanelli
\& Haynes 1985; Verdes-Montenegro et al. 2001), suggesting that the neutral gas undergoes a phase change via ionization and heating.

A key difference between compact groups and galaxy clusters is that galaxies in the cores of clusters typically have negligible star formation (Balogh et al. 1998; von der Linden et al. 2010), while some compact groups can host moderate or even intense star formation (Iglesias-Páramo \& Vílchez 1999; Gallagher et al. 2010). However, IRAS observations of compact groups indicate normal levels of thermal infrared emission, suggesting that the star formation rates (SFRs) in these groups are not generally enhanced (Allam et al. 1995; Verdes-Montenegro et al. 1998). In fact, de la Rosa et al. (2007) studied the stellar populations of elliptical galaxies in compact groups and found evidence of a mechanism that quenches star formation. This is again similar to the outer regions of clusters, which exhibit suppression of SFRs out to three virial radii (Lewis et al. 2002). Gas processing is also different in cluster cores and compact groups, where rampressure stripping does not appear to dominate gas processing (Rasmussen et al. 2008). In addition, Cortese et al. (2006) have studied a compact group falling into a cluster and found that the galaxies have undergone preprocessing due to the compact 
group environment. Despite their high densities, it appears as though the compact group environment has more in common with the outer regions of clusters than with cluster cores.

Recently, Walker et al. (2010) examined the distribution of galaxies from 12 compact groups in IRAC $(3.6-8.0 \mu \mathrm{m})$ color space and found evidence for a statistically significant gap between the location of galaxies with colors consistent with normal stellar populations and galaxies with colors indicative of star formation activity (previously identified by Johnson et al. 2007; Gallagher et al. 2008). In contrast, no gap is seen in the color-space distribution of the core of the Coma cluster, a control sample of field galaxies, or a sample of interacting galaxies. While this is not surprising, the varying distributions in IRAC color space indicate that these environments have had a significant impact on the evolution of their galaxies. However, a similar (though less pronounced) gap was observed in the outer regions of the Coma cluster. This is evidence that the gap is unique to regions of enhanced galaxy density (relative to the field) in which the neutral gas has not been fully processed.

The discovery of the gap in IRAC color space naturally leads to the question of the origin of the gap, which has been intractable owing to the small size of the previous sample. Initial results comparing the IRAC gap with specific SFRs (SSFRs; Tzanavaris et al. 2010) of the same sample of groups show that the gap in IRAC color space corresponds to a gap in SSFRs (Walker et al. 2010), suggesting a shared underlying cause. In order to better investigate the region of color space identified as the gap, we need to increase the number of observed compact group galaxies, so as to understand the characteristics of any systems that may be found in this region, as well as characterize any correlation with physical properties along the sequence in color space. In order to further populate IRAC color space, in this paper we present the colors of galaxies for a large sample of Hickson Compact Groups (HCGs; Hickson 1982) and Redshift Survey Compact Groups (RSCGs; Barton et al. 1996) at redshifts of $z<0.035$ with a full suite of IRAC data available from the Spitzer Space Telescope archive, yielding 49 compact groups including 179 galaxies.

In this paper, we adopt the following terms: the "original sample" is the 12 HCGs studied in Johnson et al. (2007) and subsequent papers. The "new sample" consists of the 21 HCGs and 16 RSCGs not previously studied by this group. The "full" or "expanded sample" is the combined original and new samples.

\section{DATA}

\subsection{Samples}

\subsubsection{Compact Groups}

Because the goal of this project is to understand galaxy evolution in the dense environment of compact groups, we began with the HCG and RSCG catalogs. These catalogs were compiled using different selection criteria, thus the groups may have slightly different characteristics; however, the RSCG criteria were chosen to create a catalog with properties similar to the HCG catalog. The HCG catalog was compiled by Hickson (1982) using photometric plates from the Palomar Sky Survey. The criteria to be classified as a compact group were $N \geqslant 4$ (four members within 3 mag of the brightest galaxy), $\theta_{N} \geqslant 3 \theta_{G}$ (no other galaxies located within a radius three times the radius containing the nuclei of the members), and $\mu_{G}<26.0$ (the surface brightness must be high). The RSCG catalog, compiled by Barton et al. (1996), utilized magnitude-limited redshift surveys to locate compact groups and determine their members.
The criteria to be a member of a compact group were to have a radial velocity difference of $\Delta V \leqslant 1000 \mathrm{~km} \mathrm{~s}^{-1}$ and galaxy separation of $D \leqslant 50 \mathrm{kpc}$ between neighbors. Galaxy groups meeting these criteria were included in the RSCG sample. There was no isolation criteria for the RSCGs, and as a result many are embedded in larger structures. There are several groups that appear in both the HCG and RSCG catalogs; naturally, these groups have only been included once in the full sample. The RSCGs include groups with three or more members. The original criteria for HCGs were four or more members, but some have been trimmed to three because one foreground or background galaxy was found to be projected onto the group (Hickson et al. 1992). Including the RSCGs in the sample will allow future analysis of the effect of varying group properties and environments on the member galaxies.

For this study, the HCG and RSCG catalogs were subselected to only include compact groups at a low enough redshift $(z<0.035)$ such that the polycyclic aromatic hydrocarbon (PAH) features do not shift out of their rest-frame bands, which would make an analysis of their distribution in color space infeasible. We searched the Spitzer archive for groups meeting this criterion and included groups for which all four channels of IRAC data are available. This yielded $21 \mathrm{HCGs}$ (in addition to the original sample of 12 HCGs) and 16 RSCGs, bringing the total number of compact groups in the full sample to 49 (see Table 1), and increasing the number of galaxies from 42 to 179 . Of these 179 galaxies, 5 are saturated in one or more IRAC bands (HCG 16d, HCG56d, HCG 90d, HCG 92b, and RSCG 4b) and are not included in the fits or statistical analysis presented in this paper. Five of the 16 RSCGs $(21,44,66,67$, and 68) are known to be embedded in larger structures. We have undertaken our analysis both with and without these groups, and in most cases, the results do not change.

\subsubsection{Comparison Samples}

As in Walker et al. (2010), we compare our compact group sample with samples of galaxies in other environments. These samples are our approximation to a "field" sample (LVL+SINGS; Dale et al. 2007, 2009), interacting galaxies (Smith et al. 2007), and two samples from the Coma cluster (the center and the infall region; Jenkins et al. 2007). See Walker et al. (2010) for a discussion of these samples.

In order to compare samples of similar characteristics, we apply a luminosity cut to our samples illustrated in Figure 1. We selected the lowest luminosity bin with more than two compact group galaxies as the minimum luminosity for all of our samples. Thus, our luminosity cut is $\log \left(L_{4.5}\left[\mathrm{erg} \mathrm{s}^{-1} \mathrm{~Hz}^{-1}\right]\right)=27.5$. Figure 2 shows the Hubble types for our compact group sample against LVL+SINGS. As can be seen in this plot, the LVL+SINGS sample is dominated by late-type galaxies (which is to be expected for the field), while the compact group sample shows a bimodal distribution of morphological types, with a large number of early-type galaxies.

\subsection{Observations}

As this study utilizes archival data, the observational setups are heterogeneous. In addition to the Spitzer data, we also utilize data from the Two Micron All Sky Survey (Skrutskie et al. 2006) to plot IR spectral energy distributions (SEDs) to shorter $\lambda$.

\subsection{Photometry}

The IRAC images were first convolved to the $8 \mu \mathrm{m}$ pointspread function in order to ensure a common pixel scale and 
Table 1

Compact Group Sample

\begin{tabular}{|c|c|c|c|c|}
\hline Group & $\begin{array}{c}\text { R.A. }^{\mathrm{a}} \\
(\mathrm{J} 2000)\end{array}$ & $\begin{array}{l}\text { Decl. }^{\mathrm{a}} \\
(\mathrm{J} 2000)\end{array}$ & $\begin{array}{c}D^{\mathrm{a}} \\
(\mathrm{Mpc})\end{array}$ & $\mathrm{AOR}(\mathrm{s})$ \\
\hline $\mathrm{H} 02$ & $00^{\mathrm{h}} 31^{\mathrm{m}} 30^{\mathrm{s}} .0$ & $+08^{\circ} 25^{\prime} 52^{\prime \prime}$ & 136.6 & r11249152,r11252224 \\
\hline H04 & $00^{\mathrm{h}} 34^{\mathrm{m}} 15^{\mathrm{s}} .9$ & $-21^{\circ} 26^{\prime} 48^{\prime \prime}$ & 108.9 & r21926400 \\
\hline $\mathrm{H} 07$ & $00^{\mathrm{h}} 39^{\mathrm{m}} 23^{\mathrm{s}} .9$ & $+00^{\circ} 52^{\prime} 41^{\prime \prime}$ & 56.4 & r11249408,r11252480 \\
\hline H15 & $02^{\mathrm{h}} 07^{\mathrm{m}} 39^{\mathrm{s}} .0$ & $+02^{\circ} 08^{\prime} 18^{\prime \prime}$ & 90.8 & r26875648,r26880512 \\
\hline H16 & $02^{\mathrm{h}} 09^{\mathrm{m}} 31^{\mathrm{s}} .3$ & $-10^{\circ} 09^{\prime} 31^{\prime \prime}$ & 52.9 & r11249664,r11252736 \\
\hline H19 & $02^{\mathrm{h}} 42^{\mathrm{m}} 45^{\mathrm{s}} .1$ & $-12^{\circ} 24^{\prime} 43^{\prime \prime}$ & 57.3 & r11249920 \\
\hline $\mathrm{H} 22$ & $03^{\mathrm{h}} 03^{\mathrm{m}} 31^{\mathrm{s}} .3$ & $-15^{\circ} 40^{\prime} 32^{\prime \prime}$ & 36.1 & r11250176,r11252992 \\
\hline $\mathrm{H} 25$ & $03^{\mathrm{h}} 20^{\mathrm{m}} 43^{\mathrm{s}} .7$ & $-01^{\circ} 03^{\prime} 07^{\prime \prime}$ & 84.5 & r26785904,r26880768 \\
\hline $\mathrm{H} 26$ & $03^{\mathrm{h}} 21^{\mathrm{m}} 54^{\mathrm{s}} .2$ & $-13^{\circ} 38^{\prime} 45^{\prime \prime}$ & 126.9 & r23027712 \\
\hline H31 & $05^{\mathrm{h}} 01^{\mathrm{m}} 38^{\mathrm{s}} .3$ & $-04^{\circ} 15^{\prime} 25^{\prime \prime}$ & 54.6 & r11250432 \\
\hline H33 & $05^{\mathrm{h}} 10^{\mathrm{m}} 47^{\mathrm{s}} .9$ & $+18^{\circ} 02^{\prime} 05^{\prime \prime}$ & 105.1 & r23027968 \\
\hline H37 & $09^{\mathrm{h}} 13^{\mathrm{m}} 35^{\mathrm{s}} \cdot 6$ & $+30^{\circ} 00^{\prime} 51^{\prime \prime}$ & 95.5 & r23028224 \\
\hline H38 & $09^{\mathrm{h}} 27^{\mathrm{m}} 38^{\mathrm{s}} .8$ & $+12^{\circ} 16^{\prime} 51^{\prime \prime}$ & 124.3 & r23026432 \\
\hline $\mathrm{H} 40$ & $09^{\mathrm{h}} 38^{\mathrm{m}} 54^{\mathrm{s}} .5$ & $-04^{\circ} 51^{\prime} 07^{\prime \prime}$ & 96.9 & r23025920 \\
\hline $\mathrm{H} 42$ & $10^{\mathrm{h}} 00^{\mathrm{m}} 21^{\mathrm{s}} .8$ & $-19^{\circ} 38^{\prime} 57^{\prime \prime}$ & 53.3 & r11250688,r11253248 \\
\hline $\mathrm{H} 47$ & $10^{\mathrm{h}} 25^{\mathrm{m}} 48^{\mathrm{s}} .4$ & $+13^{\circ} 43^{\prime} 54^{\prime \prime}$ & 135.9 & r23026688 \\
\hline $\mathrm{H} 48$ & $10^{\mathrm{h}} 37^{\mathrm{m}} 45^{\mathrm{s}} \cdot 6$ & $-27^{\circ} 04^{\prime} 50^{\prime \prime}$ & 37.7 & r11250944,r11253504 \\
\hline H54 & $11^{\mathrm{h}} 29^{\mathrm{m}} 15^{\mathrm{s}} .5$ & $+20^{\circ} 35^{\prime} 06^{\prime \prime}$ & 28.1 & r23027456 \\
\hline H56 & $11^{\mathrm{h}} 32^{\mathrm{m}} 39^{\mathrm{s}} .6$ & $+52^{\circ} 56^{\prime} 25^{\prime \prime}$ & 116.0 & r23026944 \\
\hline H57 & $11^{\mathrm{h}} 37^{\mathrm{m}} 50^{\mathrm{s}} .5$ & $+21^{\circ} 59^{\prime} 06^{\prime \prime}$ & 131.7 & r23027200 \\
\hline H59 & $11^{\mathrm{h}} 48^{\mathrm{m}} 26^{\mathrm{s}} \cdot 6$ & $+12^{\circ} 42^{\prime} 40^{\prime \prime}$ & 54.1 & r11251200 \\
\hline H61 & $12^{\mathrm{h}} 12^{\mathrm{m}} 24^{\mathrm{s}} .9$ & $+29^{\circ} 11^{\prime} 21^{\prime \prime}$ & 52.1 & r11251456 \\
\hline H62 & $12^{\mathrm{h}} 53^{\mathrm{m}} 08^{\mathrm{s}} .1$ & $-09^{\circ} 13^{\prime} 27^{\prime \prime}$ & 54.9 & r11251712 \\
\hline H67 & $13^{\mathrm{h}} 49^{\mathrm{m}} 03^{\mathrm{s}} .5$ & $-07^{\circ} 12^{\prime} 20^{\prime \prime}$ & 109.9 & r26876160,r26881024 \\
\hline H68 & $13^{\mathrm{h}} 53^{\mathrm{m}} 40^{\mathrm{s}} .9$ & $+40^{\circ} 19^{\prime} 07^{\prime \prime}$ & 40.7 & r4483584,r27600128 \\
\hline$\ldots$ & $\cdots$ & 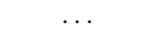 & $\ldots$ & r27600384,r27600640 \\
\hline H71 & $14^{\mathrm{h}} 11^{\mathrm{m}} 04^{\mathrm{s}} .6$ & $+25^{\circ} 29^{\prime} 06^{\prime \prime}$ & 131.8 & r23028736 \\
\hline $\mathrm{H} 79$ & $15^{\mathrm{h}} 59^{\mathrm{m}} 11^{\mathrm{s}} .9$ & $+20^{\circ} 45^{\prime} 31^{\prime \prime}$ & 68.0 & r23028992 \\
\hline H90 & $22^{\mathrm{h}} 02^{\mathrm{m}} 06^{\mathrm{s}} .0$ & $-31^{\circ} 55^{\prime} 48^{\prime \prime}$ & 32.3 & r11251968,r11253760 \\
\hline H91 & $22^{\mathrm{h}} 09^{\mathrm{m}} 10^{\mathrm{s}} .4$ & $-27^{\circ} 47^{\prime} 45^{\prime \prime}$ & 99.1 & r26877184,r26881536 \\
\hline H92 & $22^{\mathrm{h}} 35^{\mathrm{m}} 57^{\mathrm{s}} .5$ & $+33^{\circ} 57^{\prime} 36^{\prime \prime}$ & 88.6 & r6011392 \\
\hline H96 & $23^{\mathrm{h}} 27^{\mathrm{m}} 58^{\mathrm{s}} .3$ & $+08^{\circ} 46^{\prime} 27^{\prime \prime}$ & 118.5 & r12297728 \\
\hline H97 & $23^{\mathrm{h}} 47^{\mathrm{m}} 22^{\mathrm{s}} .9$ & $-02^{\circ} 19^{\prime} 34^{\prime \prime}$ & 88.3 & r26877440,r26881792 \\
\hline H100 & $00^{\mathrm{h}} 01^{\mathrm{m}} 20.8$ & $+13^{\circ} 07^{\prime} 57^{\prime \prime}$ & 71.9 & r26877696 \\
\hline R04 & $00^{\mathrm{h}} 42^{\mathrm{m}} 49^{\mathrm{s}} .5$ & $-23^{\circ} 33^{\prime} 11^{\prime \prime}$ & 89.0 & r12342784 \\
\hline R06 & $01^{\mathrm{h}} 16^{\mathrm{m}} 12^{\mathrm{s}} .4$ & $+46^{\circ} 44^{\prime} 20^{\prime \prime}$ & 69.1 & r26227712 \\
\hline $\mathrm{R} 15$ & $01^{\mathrm{h}} 52^{\mathrm{m}} 41^{\mathrm{s}} .4$ & $+36^{\circ} 08^{\prime} 46^{\prime \prime}$ & 64.3 & r18646272 \\
\hline $\mathrm{R} 17$ & $01^{\mathrm{h}} 56^{\mathrm{m}} 21^{\mathrm{s}} .8$ & $+05^{\circ} 38^{\prime} 37^{\prime \prime}$ & 75.5 & r18030336 \\
\hline $\mathrm{R} 21^{\mathrm{b}}$ & $03^{\mathrm{h}} 19^{\mathrm{m}} 36^{\mathrm{s}} \cdot 6$ & $+41^{\circ} 33^{\prime} 39^{\prime \prime}$ & 68.7 & r10483456 \\
\hline $\mathrm{R} 31$ & $09^{\mathrm{h}} 17^{\mathrm{m}} 26^{\mathrm{s}} .5$ & $+41^{\circ} 57^{\prime} 18^{\prime \prime}$ & 29.5 & r23113472,r23113728 \\
\hline $\mathrm{R} 32$ & $09^{\mathrm{h}} 19^{\mathrm{m}} 50^{\mathrm{s}} .8$ & $+33^{\circ} 46^{\prime} 17^{\prime \prime}$ & 96.3 & r4438784 \\
\hline R34 & $09^{\mathrm{h}} 43^{\mathrm{m}} 12^{\mathrm{s}} \cdot 6$ & $+31^{\circ} 54^{\prime} 44^{\prime \prime}$ & 25.7 & r4439552,r16300032 \\
\hline R38 & $10^{\mathrm{h}} 51^{\mathrm{m}} 46^{\mathrm{s}} .7$ & $+32^{\circ} 51^{\prime} 31^{\prime \prime}$ & 27.2 & r16304640 \\
\hline $\mathrm{R} 42$ & $11^{\mathrm{h}} 36^{\mathrm{m}} 51^{\mathrm{s}} 3$ & $+19^{\circ} 59^{\prime} 19^{\prime \prime}$ & 93.5 & r25498880 \\
\hline $\mathrm{R} 44^{\mathrm{b}}$ & $11^{\mathrm{h}} 44^{\mathrm{m}} 00^{\mathrm{s}} \cdot 6$ & $+19^{\circ} 56^{\prime} 44^{\prime \prime}$ & 93.6 & r3858688 \\
\hline R64 & $12^{\mathrm{h}} 41^{\mathrm{m}} 33^{\mathrm{s}} .2$ & $+26^{\circ} 03^{\prime} 56^{\prime \prime}$ & 73.7 & r10532608 \\
\hline $\mathrm{R} 66^{\mathrm{b}}$ & $12^{\mathrm{h}} 43^{\mathrm{m}} 17^{\mathrm{s}} .9$ & $+13^{\circ} 11^{\prime} 47^{\prime \prime}$ & 13.9 & r4477440,r18288896 \\
\hline $\mathrm{R} 67^{\mathrm{b}}$ & $12^{\mathrm{h}} 59^{\mathrm{m}} 32^{\mathrm{s}} .8$ & $+27^{\circ} 57^{\prime} 27^{\prime \prime}$ & 106.4 & r3859456 \\
\hline $\mathrm{R}^{\mathrm{b}} \mathrm{b}^{\mathrm{b}}$ & $13^{\mathrm{h}} 00^{\mathrm{m}} 10^{\mathrm{s}} .7$ & $+27^{\circ} 58^{\prime} 17^{\prime \prime}$ & 98.3 & r3859712,r3859968 \\
\hline R86 & $23^{\mathrm{h}} 38^{\mathrm{m}} 34^{\mathrm{s}} .4$ & $+27^{\circ} 01^{\prime} 24^{\prime \prime}$ & 117.6 & r10926592 \\
\hline
\end{tabular}

Notes.

${ }^{a}$ Group R.A., decl., and distance (corrected for Virgo+GA+Shapley) taken from NED. The cosmology used was $H_{0}=73 \mathrm{~km} \mathrm{~s}^{-1} \mathrm{Mpc}^{-1}, \Omega_{\text {matter }}=0.27$, and $\Omega_{\text {vacuum }}=0.73$.

b RSCGs known to be embedded in larger structures.

resolution, and then photometry was performed using SURPHOT (Reines et al. 2008). The program determines apertures by finding a specified contour level in a reference image, and then measuring the flux within the same aperture for each image. The apertures were determined using a combined image of all four IRAC bands (weighted by $\lambda^{-1}$ ), using a contour level of $1-2 \sigma$. Several background annuli were used, with inner radii ranging from 2 to $2.5 \times$ the radius of the aperture and outer radii ranging from 2.5 to $3 \times$ the radius of the aperture, using both the mode and resistant mean. Uncertainties were calculated from the standard deviation of the fluxes measured using different backgrounds. Extended source aperture corrections were made using the algorithms of T. Jarrett, ${ }^{6}$ and average corrections were $4 \%$, $2 \%, 9 \%$, and $14 \%$ at $3.6,4.5,5.8$, and $8.0 \mu \mathrm{m}$, respectively. There are slight differences from Johnson et al. (2007), likely due to variations in background measurements, though the new data are still consistent with the previous measurements.

\section{COLOR SPACE}

\subsection{Full Sample}

The IRAC colors of the full sample are presented in Figure 3, which shows that an underdensity of galaxies is persistent, though it has become more akin to a canyon than a gap. As the sample is now composed of 179 galaxies, there are more galaxies with colors that place them within the canyon, though there is still a dearth of galaxies in this region relative to the number of galaxies with MIR-blue and MIR-red colors. The plot in Figure 3 shows the data with a linear fit; however, a new result seen in this full sample of 49 compact groups is the curvature of the distribution in color space, which will be discussed in Section 6.4. To quantify this curvature, we fit a quadratic to the data, also shown in Figure 3. Thus, we can undertake our analysis with respect to the line or the curve, both fit to the data.

For the statistical analysis, we considered the MIR colors in two ways: rotated so that the line shown in Figure 3 became the axis, and unwrapped so that the curve shown in Figure 3 became the axis. For both methods, the sample was shifted to have a mean value of zero. We have redefined the gap as the canyon using both sets of colors, as illustrated in Figure 4. In both plots, we define the canyon to be where the histogram is less than half its median value and $C_{\mathrm{MIR}}$ is $<1.0$. The bounds of the canyon are indicated by the vertical dotted lines. The blue side of the canyon changes depending on whether we define it using the rotated or unwrapped color - the canyon determined from the unwrapped data is slightly smaller. However, the results of our statistical analysis do not change.

The plots in Figure 5 show the results of the Kolmogorov-Smirnov (K-S) test comparing the rotated and unwrapped color distributions with a model of a uniform distribution, where $D$ is the maximum difference between the sample and the model, and $\alpha$ gives the probability that the model matches the data. While we do not expect any particular sample to have a uniform distribution, using a model of a uniform distribution as a baseline facilitates comparisons between environments. The gray region indicates the canyon, which is clearly manifested in this plot as the flat portion of the cumulative distribution function (CDF). The canyon is less extended in this larger data set than the gap seen in the original sample, but it is still present, and $\alpha$ is small enough that we can conclusively state that the compact group sample is not drawn from a uniform distribution in color space. The two methods of unraveling the MIR colors yield the same result in the K-S test. Further analysis uses the unwrapped colors, because the canyon galaxies as defined by the unwrapped sample are more cleanly defined in color space (shown in Figure 6). Excluding the five RSCGs that are embedded in larger structures $(21,44,66,67$, and 68) increases $\alpha$ by an order of magnitude but does not change the result.

\footnotetext{
6 http://web.ipac.caltech.edu/staff/jarrett/irac/calibration/
} 


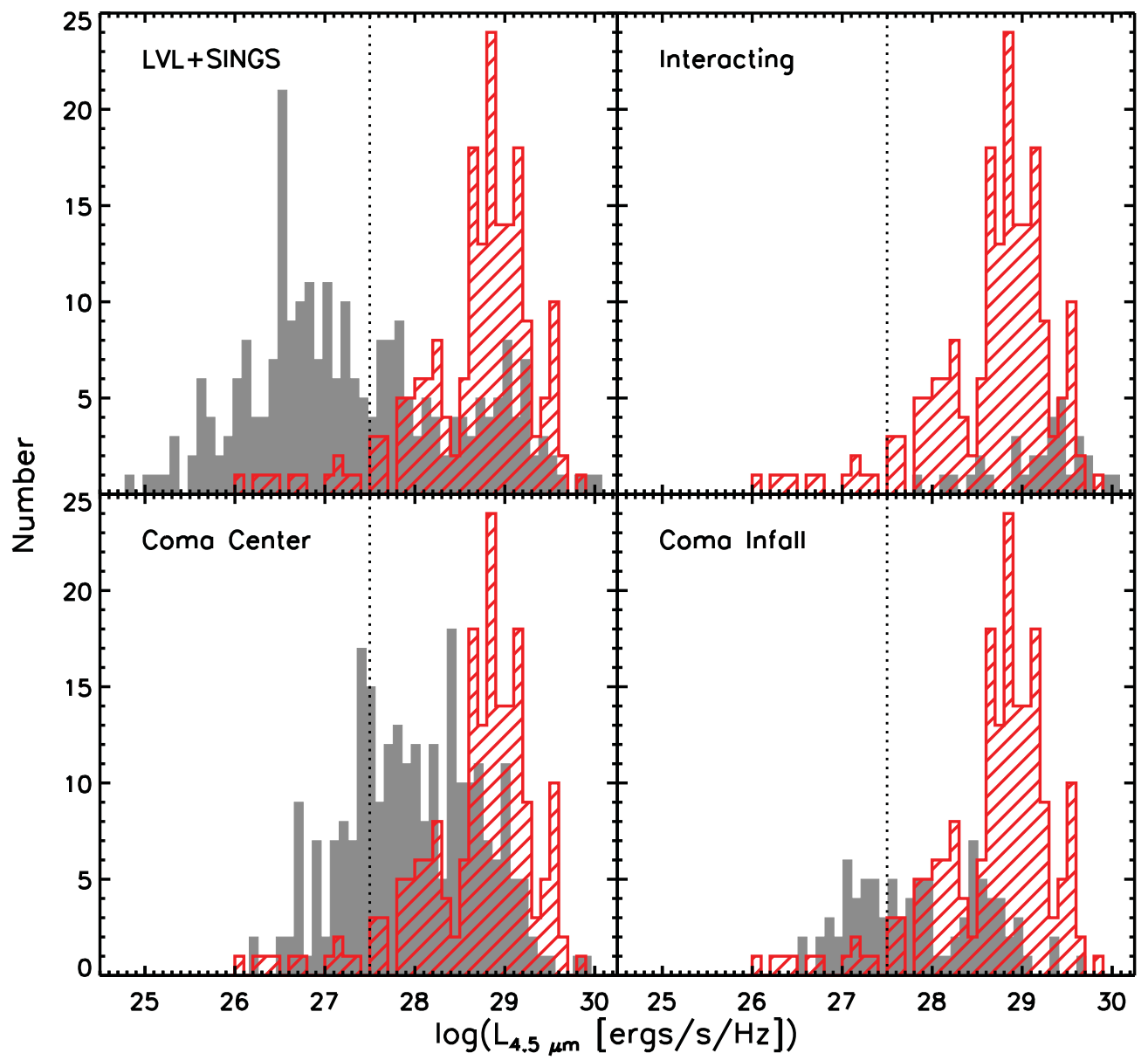

Figure 1. Histograms of $L_{4.5}$ for the compact group sample (striped) overlaid on the comparison samples (solid). The dotted vertical line indicates the luminosity cut. (A color version of this figure is available in the online journal.)

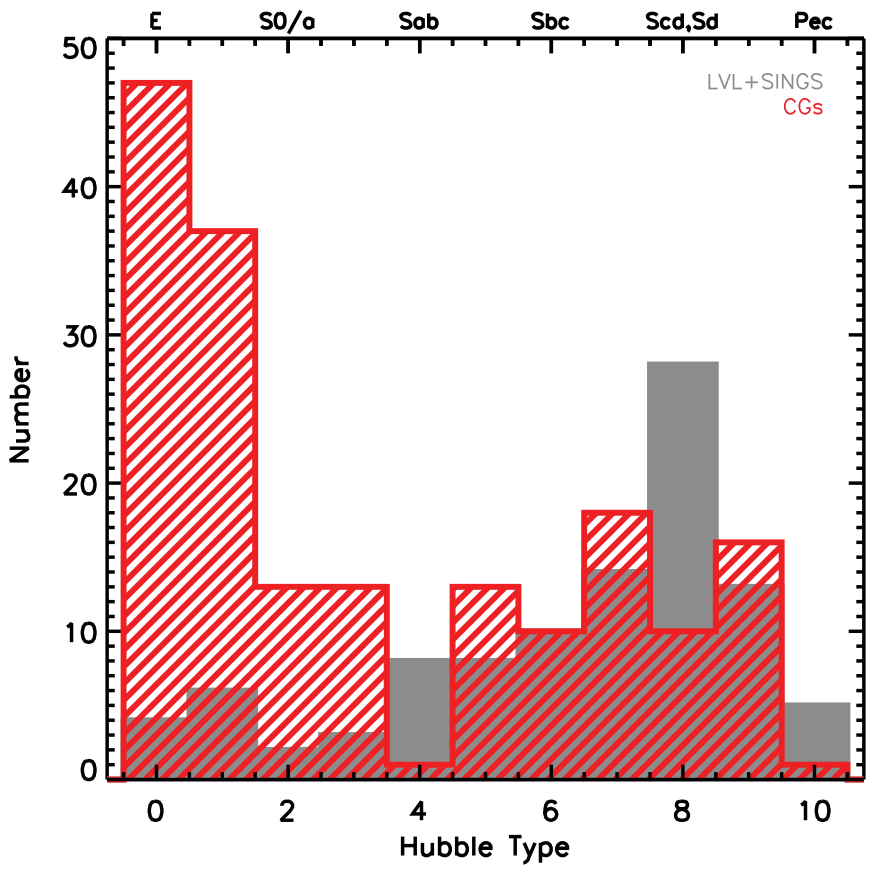

Figure 2. Distribution of morphological types (following Haynes \& Giovanelli 1984) for the compact group galaxies (striped) and LVL+SINGS galaxies (solid). As expected for a field sample, the LVL+SINGS galaxies are dominated by late types, while the compact group sample has a bimodal distribution.

(A color version of this figure is available in the online journal.)

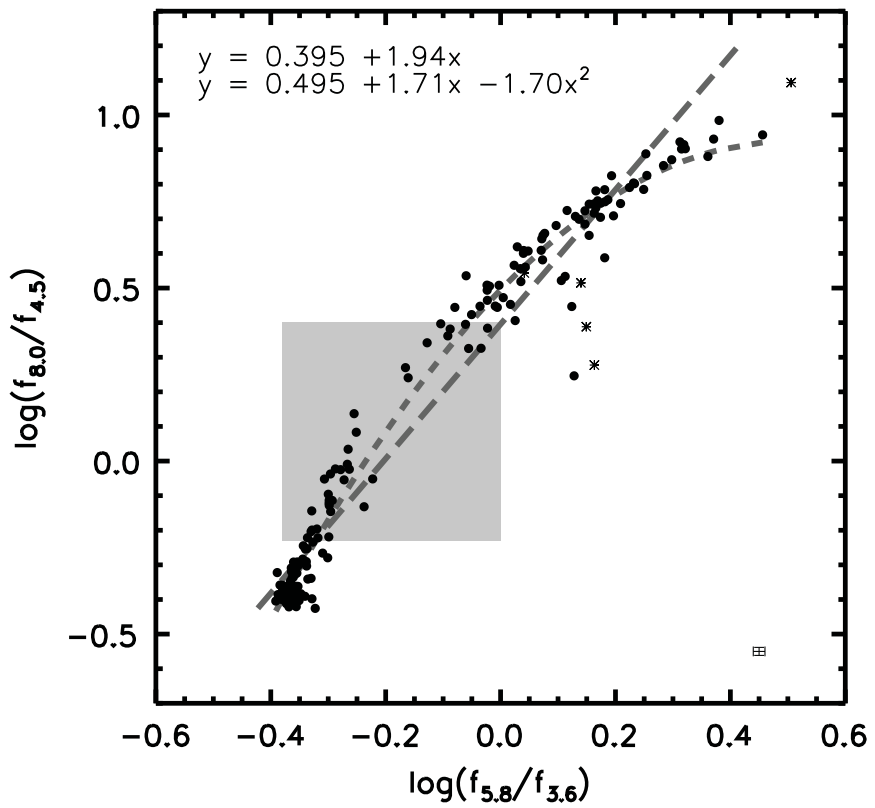

Figure 3. Color-space distribution of the 179 compact group galaxies constituting the full sample; the shaded box highlights the location of the gap in the original sample (Walker et al. 2010). The asterisks indicate galaxies that are mildly saturated in one or more IRAC channels; these five galaxies are not included in further analysis. We performed two different fits to the data, as shown above. 

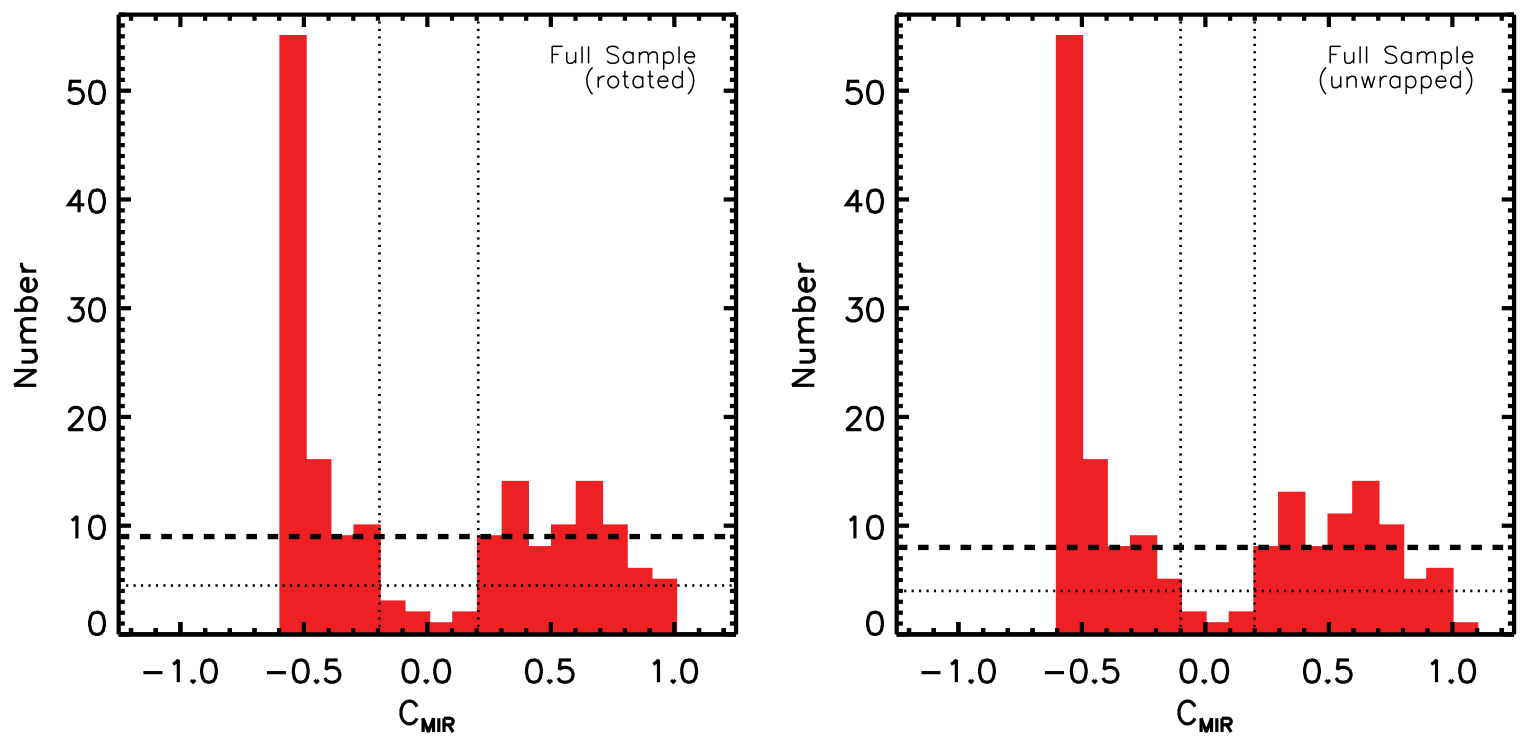

Figure 4. Rotated color-space distribution (left) and unwrapped color-space distribution (right) as described in Section 3.1. In both plots, the dashed line indicates the median, and the horizontal dotted line is half that value. The vertical dotted lines indicate the boundaries of the canyon, defined to be where the distribution is less than half the median value with an MIR color $<1.0$. In the rotated sample, $5 \%$ of the galaxies fall in the canyon, while for the unwrapped sample, $3 \%$ of galaxies fall in the canyon.

(A color version of this figure is available in the online journal.)
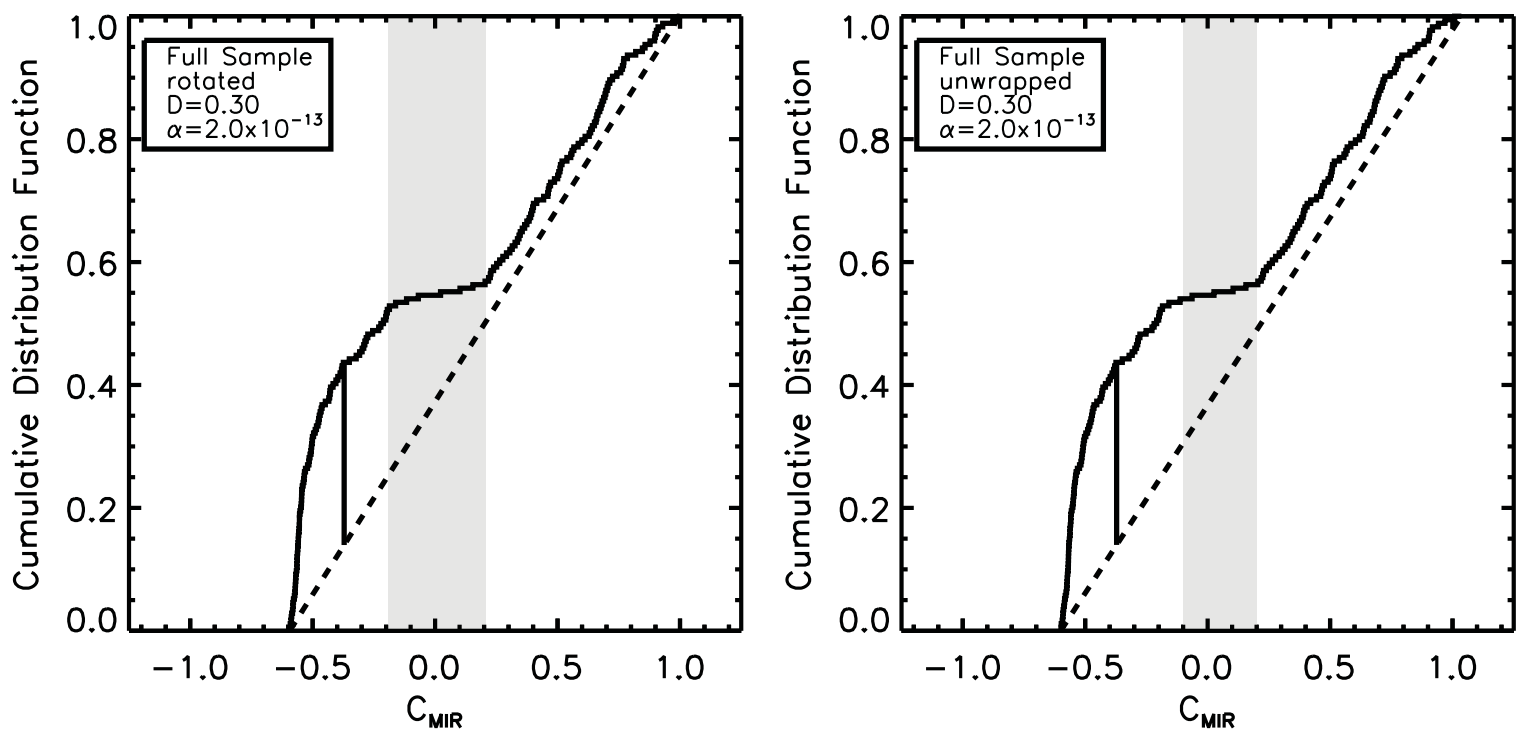

Figure 5. K-S test for the left: rotated color-space distribution; right: unwrapped color-space distribution of the full sample against a model of uniform distribution. The maximum deviation $D$ of the sample from the model is indicated by the vertical line. This large value of $D$ means a low probability that the compact groups are drawn from a uniform distribution, $2 \times 10^{-11} \%$.

\subsection{Subsamples}

To investigate how the compact group environment affects galaxy evolution, we investigated the color-space distribution of various subsamples. If a clear trend with physical properties were found, it would provide a clear picture of how environment affects galaxy evolution in compact groups.

\subsubsection{Original versus New}

To ensure that the properties of the expanded sample are consistent with the original sample, we compare it with the original sample. The CDFs of the new and original sample are shown on the right in Figure 6, which illustrates that the new sample is consistent with the original sample. The difference in the gap seen in the original sample and the canyon defined by the full sample (highlighted by the shaded region) is clearly illustrated by the different ranges of the flat portion of the $\mathrm{CDF}$ - the canyon is narrower and slightly less pronounced than the original gap.

\subsubsection{HCGs versus RSCGs}

To determine whether the different selection criteria of the HCG and RSCG catalogs find groups with different properties, we compare groups from the two catalogs. The color-space distribution of the HCG galaxies and RSCG galaxies is shown in Figure 7. We see that the HCGs and RSCGs occupy color space very similarly. 

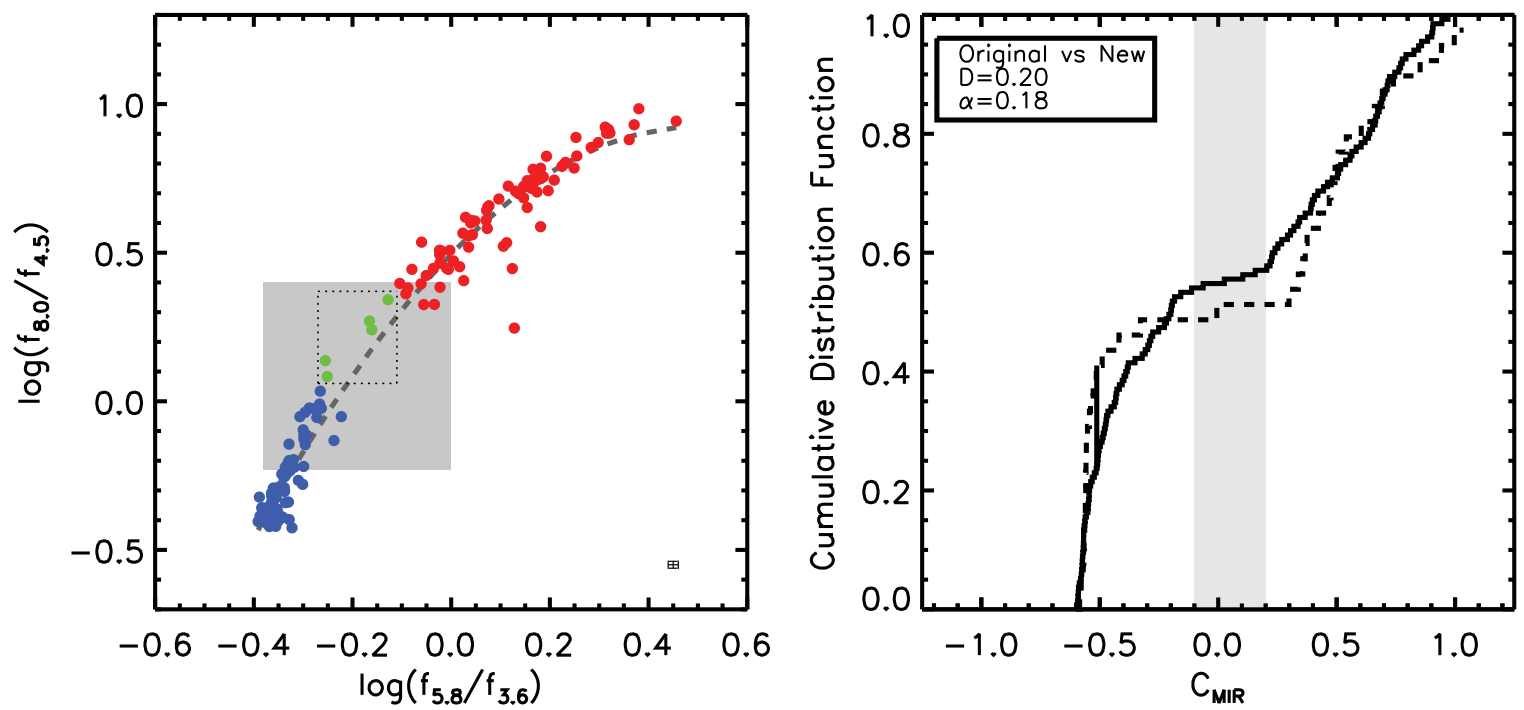

Figure 6. Left: the color-space distribution of the compact group galaxies, with symbol color illustrating whether a galaxy is blueward (blue), in (green), or redward (red) of the gap. Right: K-S test comparing the original sample of 12 HCGs (dashed line) with the new sample of 37 compact groups (solid line). These two samples are very similar, with a fairly high probability of being drawn from the same parent distribution, $18 \%$.
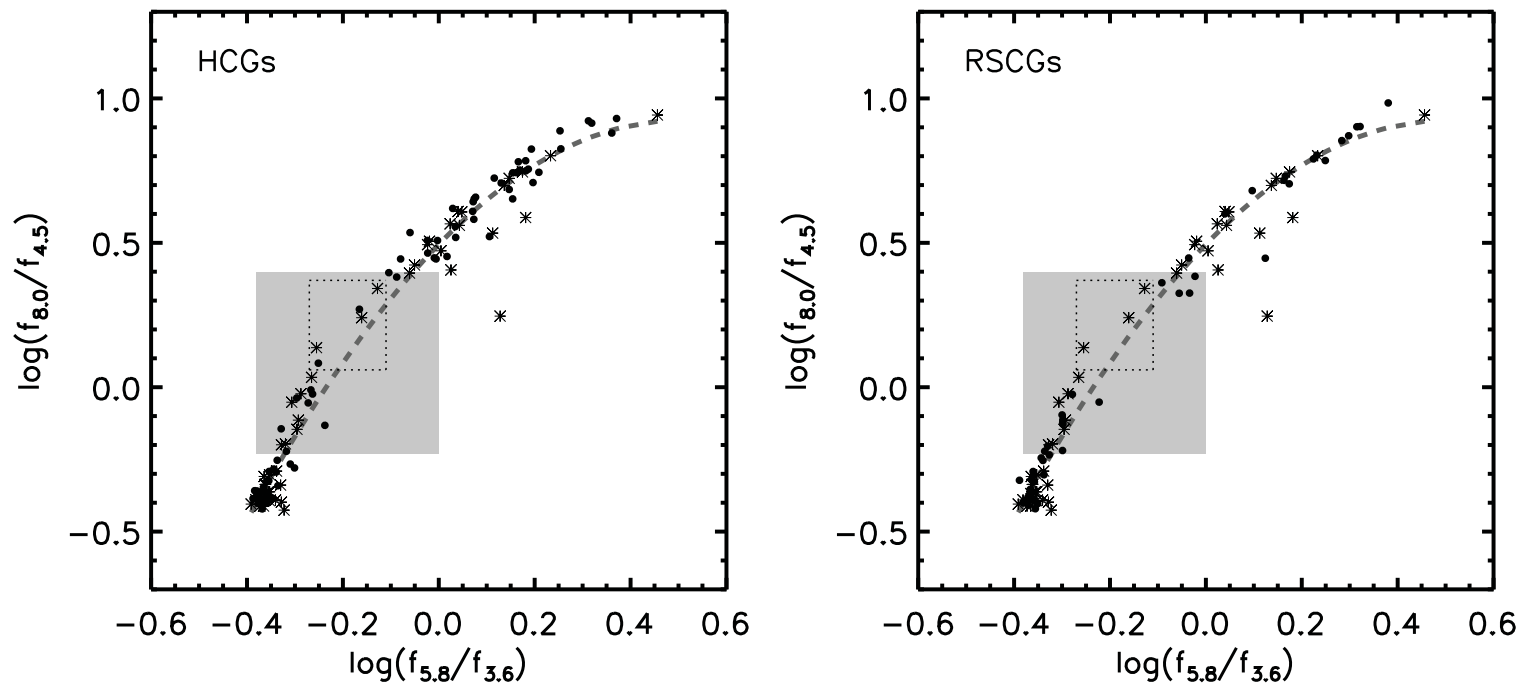

Figure 7. Left: the color-space distribution of the 124 HCG galaxies; right: the 50 RSCG galaxies.

Table 2

Results of Statistical Analysis

\begin{tabular}{lccccc}
\hline \hline Compact Groups vs. & $N$ & $N_{\text {CGs }}$ & $D$ & $\alpha$ & Consistent? \\
\hline Uniform distribution & $\ldots$ & 174 & 0.30 & $2.0 \times 10^{-13}$ & Reject \\
LVL+SINGS & 93 & 174 & 0.47 & $3.8 \times 10^{-12}$ & Reject \\
Interacting & 31 & 78 & 0.41 & $1.6 \times 10^{-3}$ & Reject \\
Coma center & 114 & 173 & 0.36 & $6.0 \times 10^{-8}$ & Reject \\
Coma infall & 47 & 173 & 0.18 & 0.16 & Do not reject
\end{tabular}

Notes. Results of the K-S test for the compact group distribution against a model and the comparison samples, unwrapped using the quadratic fit to the compact group distribution. $D$ is the maximum deviation (possible range: $0-1$ ) between the two CDFs, while $\alpha$ gives the probability that the two samples were drawn from the same parent distribution (which depends on the number of galaxies in the two samples, $N$ and $N_{\mathrm{CGs}}$ ). Based on $\alpha$, we have determined whether the two samples are consistent.

\subsubsection{Separated by Physical Properties}

As compact group galaxies occupy color space differently than galaxies in other environments (shown in Table 2 and Section 3.3, as well as Walker et al. 2010), we expect to see a trend with physical properties of compact groups, especially the properties that differentiate them from other environments. Thus, we binned the groups by projected physical diameter and projected physical number density and examined color space as a function of these properties, as shown in Figure 8. Both of these properties could be an indicator of how frequently or intensely interactions occur, which could plausibly affect the triggering of star formation and/or transformation from activity to quiescence. However, as these figures show, there does not seem to be any trend with these properties. Further investigation of the CDFs of the subsamples reveals that the shape of the subsample's CDF does not correlate with either of these properties. This will be discussed further in Section 6.2.

\subsection{Comparison Samples}

The color-space distributions of our comparison samples are shown in Figure 9. As this figure illustrates, the LVL+SINGS sample spans almost the exact same region of color space as the compact group sample, though there is no evidence for a canyon. The interacting sample does not occupy the blue region 

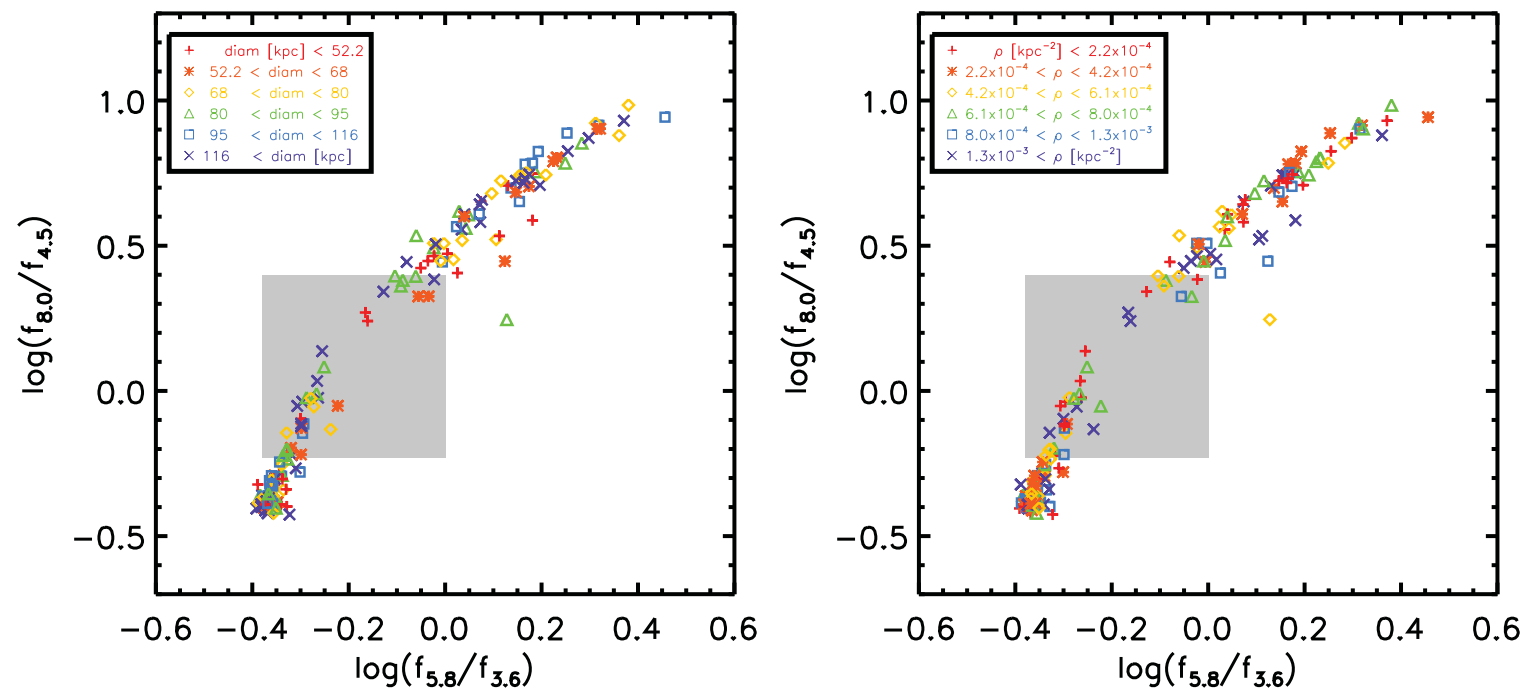

Figure 8. Color-space distribution of the full sample, broken into subsamples based on (left) projected physical diameter and (right) projected physical density. Neither of these plots shows a trend, indicating that these parameters do not play a key role in determining a galaxy's location in color space within the range represented by the compact group sample.

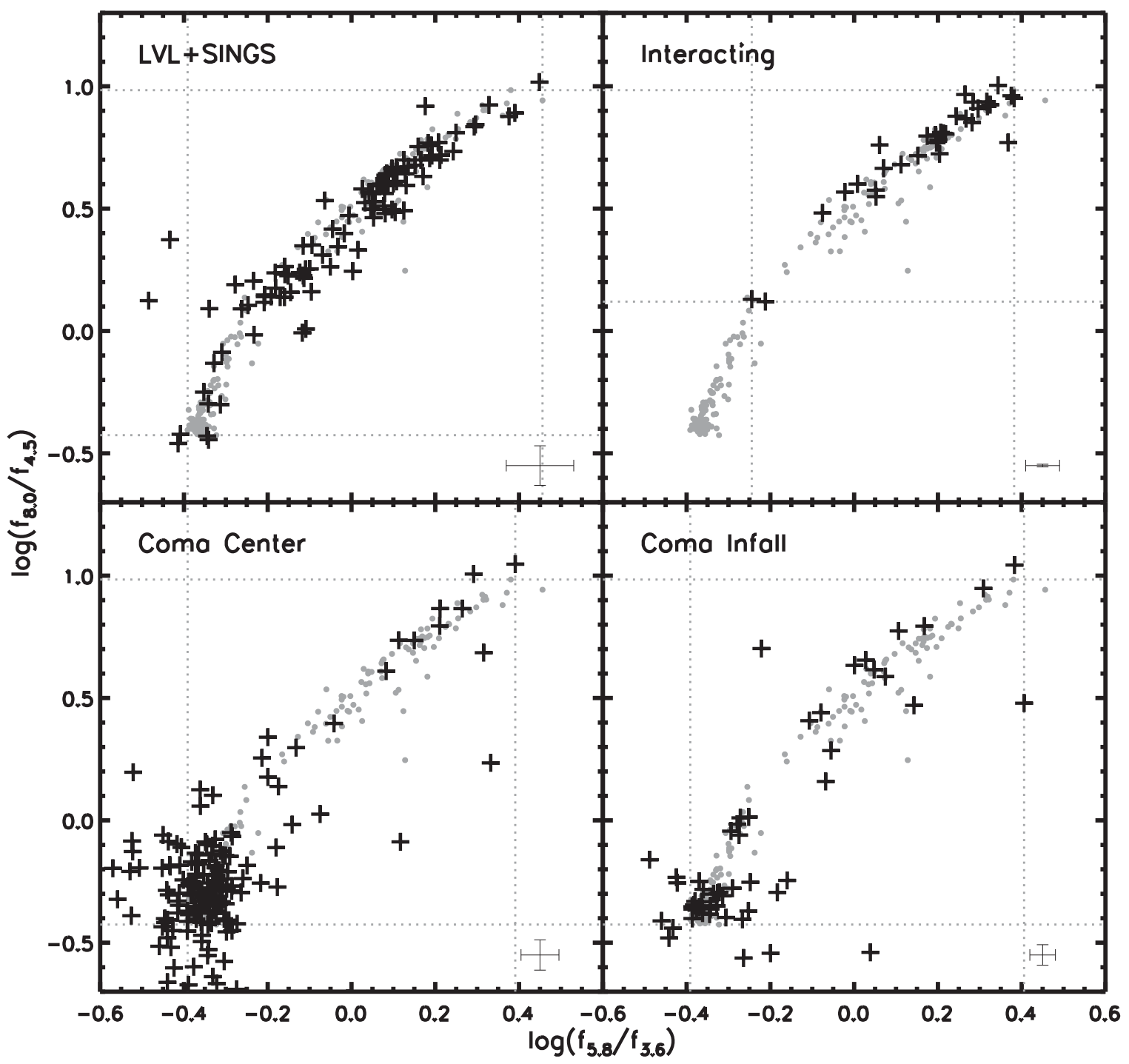

Figure 9. Color-space distribution of the comparison samples (black plus signs) overlaid on the compact group sample (gray dots). 

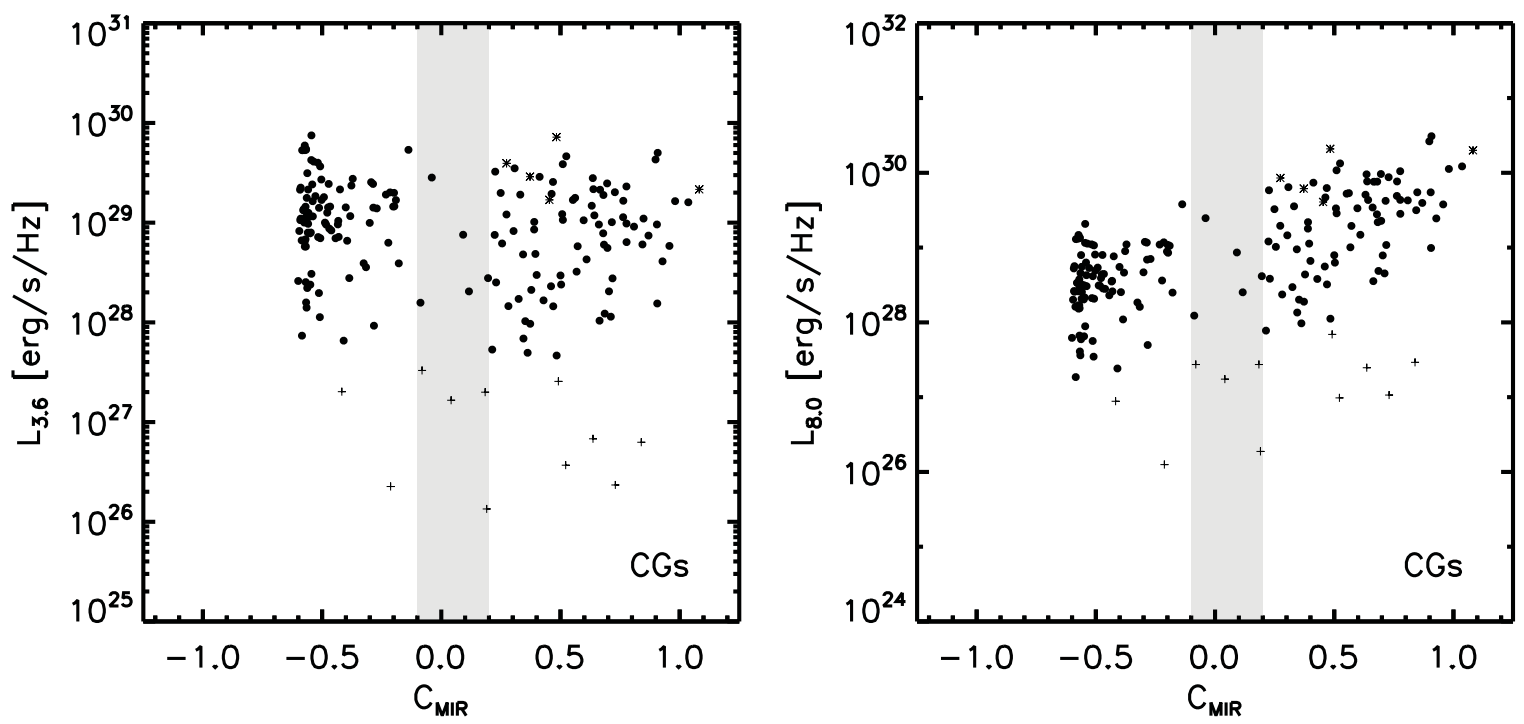

Figure 10. $L_{3.6}$ (left) and $L_{8.0}$ (right) color-magnitude diagrams for compact group galaxies. The plus signs represent galaxies below the luminosity cut (as discussed in Section 2.1.2 and Figure 1); the asterisks indicate saturated galaxies. The shaded region indicates the canyon in IRAC color space.
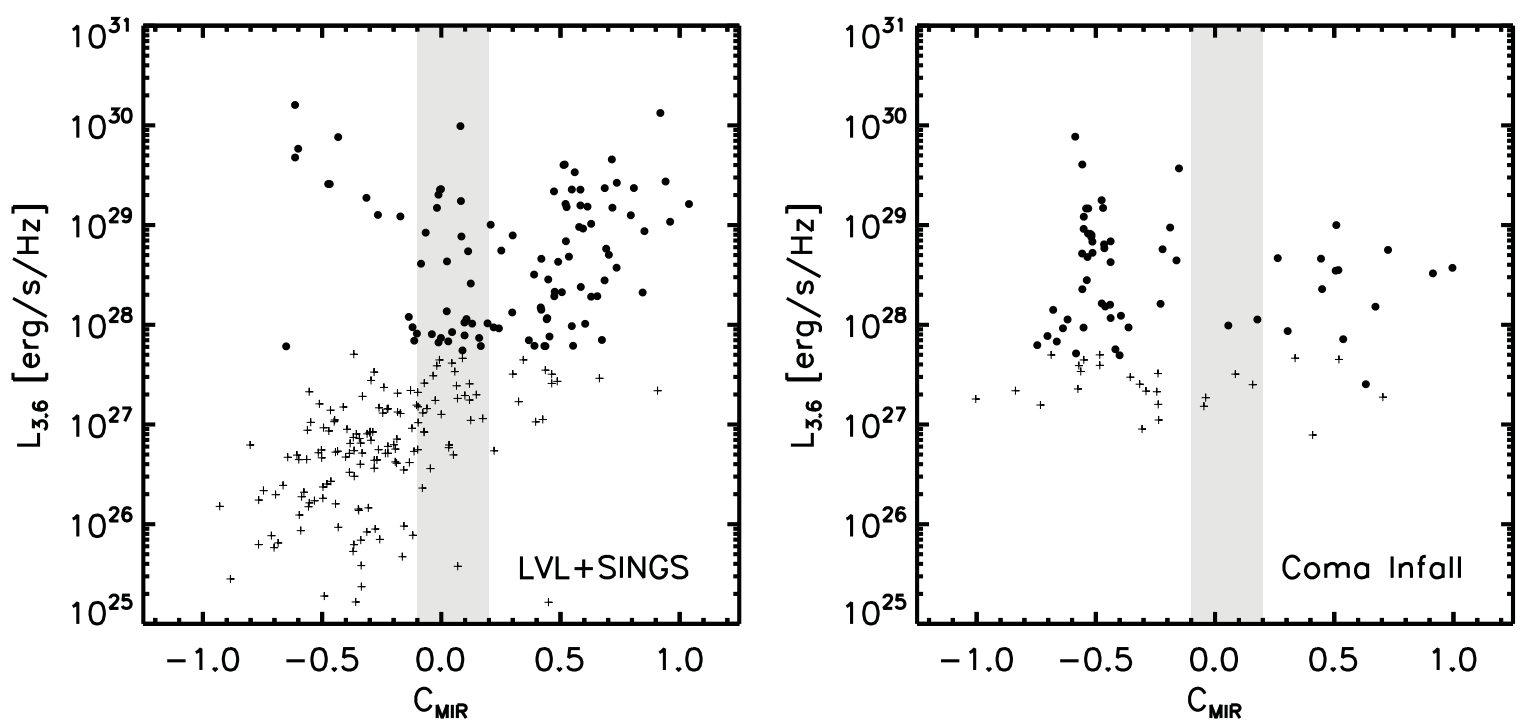

Figure 11. $L_{3.6}$ color-magnitude diagrams of galaxies in (left) the field sample of LVL+SINGS and (right) the infall region of the Coma cluster. The plus signs represent galaxies below the luminosity cut (as discussed in Section 2.1.2 and Figure 1). The shaded region indicates the canyon in IRAC color space.

of color space, indicating that this sample does not contain any galaxies dominated by stellar emission. In contrast, galaxies in the center of the Coma cluster predominantly fall in the blue region of color space, with only a few galaxies whose colors indicate activity. Like the Coma center, the infall region of the Coma cluster shows a concentration of galaxies with normal stellar colors but also contains galaxies with colors indicative of activity and exhibits an underdensity of points in the same region as the canyon in the compact group sample. We performed twodistribution $\mathrm{K}-\mathrm{S}$ tests comparing these samples with the compact group sample; the results are given in Table 2.

It is important to consider the color-space distributions of the samples in the context of the morphologies of their galaxies, given in Figure 2 for the compact group and field samples. As discussed in Walker et al. (2010), untangling the effects of morphology on MIR color is non-trivial. To some extent, morphology and MIR color are expected to track each other; e.g., late-type spirals are typically star forming and therefore expected to have red MIR colors. Therefore, it could be the case that the different morphological types that dominate each of the comparison samples are driving the K-S test results. For example, the interacting galaxy sample has no E/S0 galaxies by selection, and the morphology-density relation means that primarily quiescent E/S0s are found in the Coma core sample. Unlike these two samples, both the compact group and LVL+SINGS samples span the range of morphological types. While MIR color tracks morphology quite well in the compact group sample, this is not the case for the LVL+SINGS galaxies (Walker et al. 2010). Thus, while the dearths seen in both color-space and morphological distribution for the compact group sample may be caused by the same evolutionary process, the causal relationship is unclear.

\section{COLOR-MAGNITUDE DIAGRAMS}

Galaxy evolution is tied to galaxy size and buildup. If the compact group galaxies show a trend with mass or activity, this would be revealed in a color-magnitude diagram (CMD). Comparison of the compact group CMD with LVL+SINGS and Coma Infall (shown in Figures 10-12) provide insight 

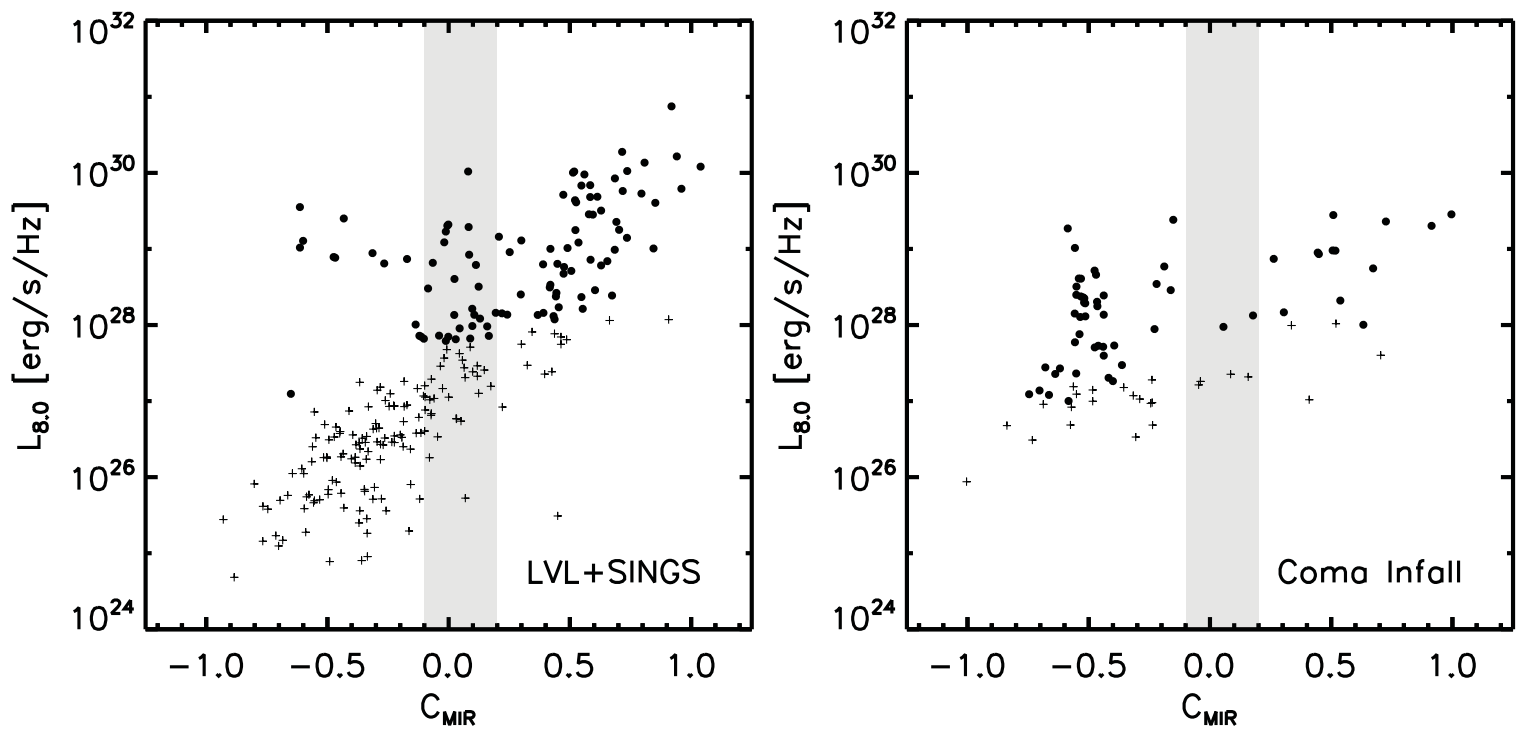

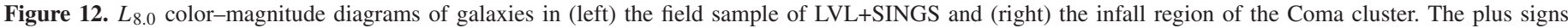
represent galaxies below the luminosity cut (as discussed in Section 2.1.2 and Figure 1). The shaded region indicates the canyon in IRAC color space.
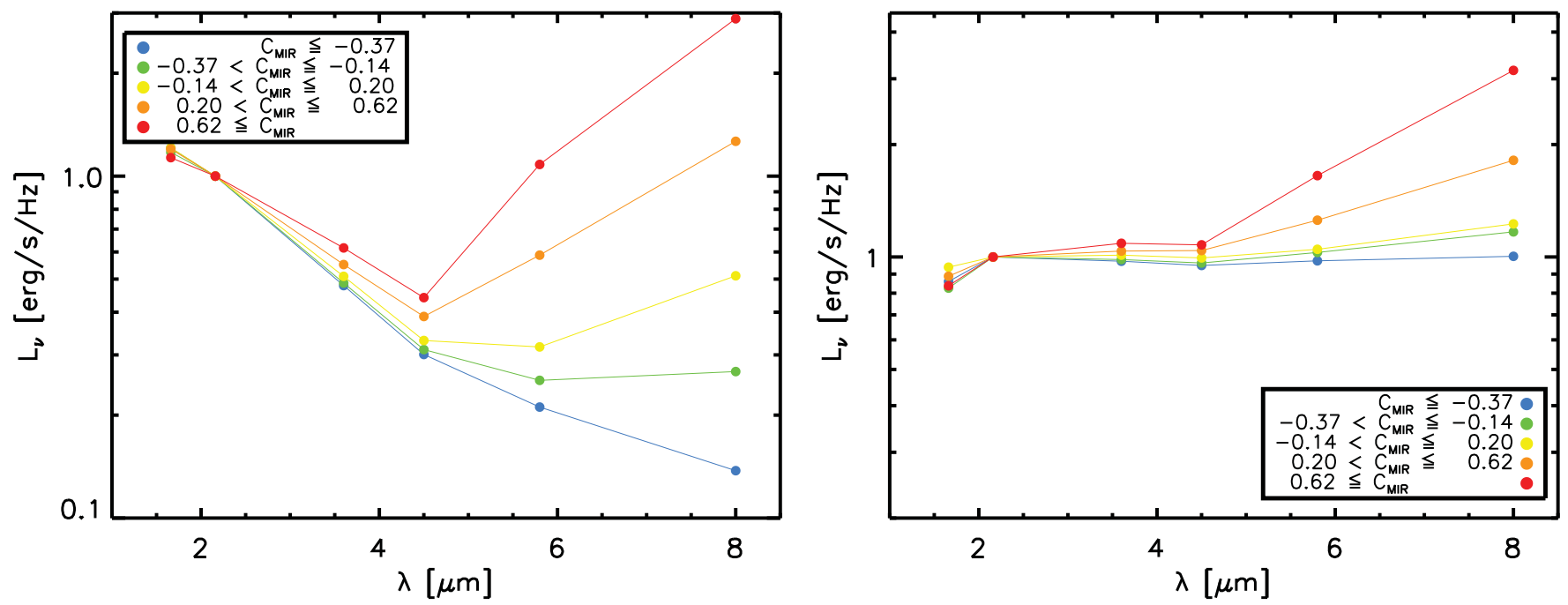

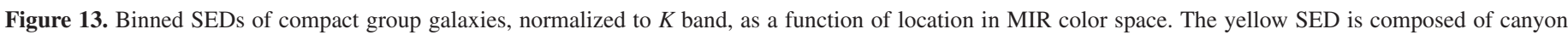

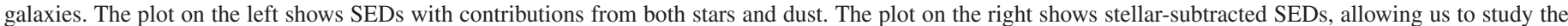
dust.

into galaxy properties. All three samples show a trend for MIR-red galaxies to be more luminous. This is not surprising, as we would expect that galaxies with activity would have more warm dust and PAH emission, thus increasing their $L_{8.0}$. One notable feature is the dearth of intermediate-luminosity, MIR-blue galaxies in the field sample, though this could be a selection effect, specifically that the SINGS sample was chosen to include "interesting" galaxies, and thus mid-luminosity, MIRblue galaxies may not have been included.

\section{SPECTRAL ENERGY DISTRIBUTIONS}

In order to investigate the relative contributions of amount and temperature of dust in compact group galaxies as a function of color space, we have binned SEDs of individual galaxies as a function of location in color space, shown on the left in Figure 13. Since we are interested in the ISM, we extracted the contribution from stellar light by scaling a $5000 \mathrm{~K}$ blackbody to the $K$-band emission. If the dust properties (e.g., temperature, composition, excitation) are the same in galaxies across color space, and the variation in MIR color is simply due to different amounts of dust and PAH emission, we expect the slope of the stellar-subtracted SEDs to be the same regardless of location in color space. If the different MIR colors are in fact dominated by varying dust properties, we expect the slope of the stellar-subtracted SEDs to change as a function of MIR color. As can be seen on the right in Figure 13, the stellar-subtracted SEDs do have clearly different slopes as a function of MIR color, which means that the range of MIR colors we see in the compact group sample is not simply due to varying amounts of dust between galaxies. An analysis of the LVL+SINGS SEDs yields a similar result. Thus, the trend in IRAC color space can be attributed to the properties of dust in the galaxies, and the canyon reflects a range of moderate dust properties.

\section{DISCUSSION}

We have compiled a full sample of 49 compact groups composed of 33 HCGs and 16 RSCGs. We see that the deficit (previously identified as a gap, see Section 1) between galaxies 
with colors consistent with normal stellar emission (MIR blue) and galaxies with colors indicative of activity (MIR red) is persistent in the larger sample. We investigate physical trends as a function of color space and look at the structure of the color-space distribution. We also compare the compact group color-space distribution with samples of galaxies in other environments. We consider the SEDs of the galaxies to assess whether dust quantity or temperature changes as a function of color space.

\subsection{The Canyon}

The gap seen in the original sample of 12 HCGs (Johnson et al. 2007; Walker et al. 2010) needs to be redefined in terms of the full sample presented here. While there is still an underdensity between galaxies whose colors are consistent with normal stellar populations and galaxies whose colors are indicative of activity, it is no longer the clear-cut gap seen originally. Now with better statistics, it is sparsely populated and thus more of a canyon. As shown in Figure 4, we quantified the location of the canyon via a histogram of the color-space distribution. As the full sample has $\sim 4 \times$ more galaxies than the original sample, there is naturally a larger absolute number of galaxies in the canyon, though the relative number of galaxies is comparable $(2.4 \%$ of galaxies from the original sample, $2.8 \%$ of galaxies from the expanded sample). The larger number of galaxies in this region will allow us to learn about properties of galaxies that exhibit these intermediate colors.

\subsection{Impact of Subsamples}

The larger number of galaxies available in the full sample allows us to look for trends in color space. Comparing the HCGs with the RSCGs shows that they are consistent with being drawn from the same parent distribution. Breaking the full sample into subsamples based on projected physical diameter or density does not reveal any trends in color space. This is a puzzling result-we hypothesize that compact groups are quantitatively different from other samples in MIR color space owing to our analysis of the compact group and comparison samples, yet we do not see any trends with these properties within the compact group sample. It appears that projected physical diameter and density are not the dominant properties in determining the colorspace location of compact group galaxies over the parameter ranges of this sample. It may simply be that the compact group environment is pre-selected to be compact and dense, and thus does not have a large enough variation in group diameters or densities to reveal trends in color space. However, Johnson et al. (2007) found that the original sample exhibits a trend in color space with group H I richness, so this seems to be the crucial property in color-space distribution. Regardless of a group's diameter or density, star formation relies on the presence of cold gas.

\subsection{Environment}

In agreement with previous work (Walker et al. 2010), the color-space distribution of the compact group sample is statistically different from a control sample of "field" galaxies, a sample of interacting galaxies, and galaxies from the center of the Coma cluster. However, we cannot rule out the hypothesis that the compact group sample is drawn from the same parent distribution as the sample from the infall region of the Coma cluster. This supports the hypothesis that there is something special about this environment-one of high galaxy density where the neutral gas has not typically been fully preprocessed (meaning stripped, ionized, expelled, or otherwise modified through a galaxy interaction process). M. Cluver et al. (2012, in preparation) found that galaxies in or near the canyon show anomalous $\mathrm{H}_{2}$ excitation, similar to that seen in the shock in Stephan's Quintet (HCG 92; Cluver et al. 2010). This could be related to a rapid end to star formation in compact group galaxies, leading to the canyon observed in IRAC color space.

$$
\text { 6.4. SEDs }
$$

The full sample revealed a curvature in color space not apparent in the original sample that was sparsely populated. The changing shape of the dust-subtracted SEDs as a function of MIR color indicates that the colors are not dominated solely by varying amounts of dust within the galaxies. Rather, the dust temperature distribution and/or PAH contributions change as a function of MIR color. We are unable to determine dust temperatures for these galaxies because the IRAC bands contain significant PAH features, so we cannot disentangle the PAH emission from the dust temperature, and since the SEDs are not turning over, we do not cover the peak of the emission. We have looked at IRS spectra of compact group galaxies in different regions of IRAC color space and found that the spectra of the bluest galaxies decrease with wavelength and show no $\mathrm{PAH}$ features. The PAH features then appear fairly quickly as you consider redder galaxies. They seem to be fully formed by the canyon region and do not change significantly as you look redward. The primary change redward of the canyon seems to be the slope of the spectra (L. M. Walker et al. 2012, in preparation). This suggests that galaxies undergoing activity have different dust compositions than quiescent galaxies.

\subsection{Conclusions}

We have determined that the distribution of compact group galaxies in MIR color space still shows an underdensity in the canyon region of color space. This distribution is most similar to that of the Coma infall region, implying a similarity in environment. The distribution of colors is caused by varying dust temperatures and PAH emission rather than varying amounts of dust. However, there are still many unexplained properties of this expanded sample.

K.E.J. gratefully acknowledges support for this paper provided by NSF through CAREER award 0548103 and the David and Lucile Packard Foundation through a Packard Fellowship. S.C.G. thanks the National Science and Engineering Research Council of Canada and The Ontario Early Researcher Award Program for support. J.C.C. was supported by the National Science Foundation through award 0908984. L.M.W thanks David Whelan for helpful discussions. We also thank the anonymous referee for their constructive comments. This research has made use of the NASA/IPAC Extragalactic Database (NED), which is operated by the Jet Propulsion Laboratory, California Institute of Technology, under contract with the National Aeronautics and Space Administration.

Facility: Spitzer (IRAC)

\section{REFERENCES}

Allam, S., Assendorp, R., Longo, G., \& Richter, G. 1995, Planet. Space Sci., 43, 1371

Balogh, M., Schade, D., Morris, S., et al. 1998, ApJ, 504, 75

Barton, E., Geller, M., Ramella, M., Marzke, R., \& da Costa, L. 1996, AJ, 112 , 871 
Cluver, M., Appleton, P. N., Boulanger, F., et al. 2010, ApJ, 710, 248

Cortese, L., Gavazzi, G., Boselli, A., et al. 2006, A\&A, 453, 847

Dale, D., Gil de Paz, A., Gordon, K. D., et al. 2007, ApJ, 655, 863

Dale, D. A., Cohen, S. A., Johnson, L. C., et al. 2009, ApJ, 703, 517

de la Rosa, I., de Carvalho, R., Vazdekis, A., \& Barbuy, B. 2009, AJ, 133, 330

Gallagher, S., Durrell, P. R., Elmegreen, D. M., et al. 2010, AJ, 139, 545

Gallagher, S., Johnson, K., Hornschemeier, A., Charlton, J., \& Hibbard, J. 2008, ApJ, 673, 730

Giovanelli, R., \& Haynes, M. 1985, AJ, 292, 404

Haynes, M., \& Giovanelli, R. 1984, AJ, 89, 758

Hickson, P. 1982, ApJ, 255, 382

Hickson, P., Mendes de Oliveira, C., Huchra, J., \& Palumbo, G. 1992, ApJ, 399, 353

Iglesias-Páramo, J., \& Vílchez, J. 1999, ApJ, 518, 94

Jenkins, L., Hornschemeier, A., Mobasher, B., Alexander, D., \& Bauer, F. 2007, ApJ, 666, 846
Johnson, K. E., Hibbard, J. E., Gallagher, S. C., et al. 2007, AJ, 134, 1522

Lewis, I., Balogh, M., De Propris, R., et al. 2002, MNRAS, 334, 673

Mulchaey, J. 2002, ARA\&A, 38, 289

Rasmussen, J., Ponman, T., Verdes-Montenegro, L., Yum, M., \& Borthakur, S. 2008, MNRAS, 388, 1245

Reines, A. E., Johnson, K. E., \& Goss, W. M. 2008, AJ, 135, 2222

Skrutskie, M. F., Cutri, R. M., Stiening, R., et al. 2006, AJ, 131, 1163

Smith, B., Struck, C., Hancock, M., et al. 2007, AJ, 133, 791

Tzanavaris, P., Hornschemeier, A. E., Gallagher, S. C., et al. 2010, ApJ, 716, 556

Verdes-Montenegro, L., Yun, M., Perea, J., del Olmo, A., \& Ho, P. 1998, ApJ, 497, 89

Verdes-Montenegro, L., Yun, M., Williams, B., et al. 2001, A\&A, 377, 812

von der Linden, A., Wild, V., Kauffmann, G., White, S., \& Weinmann, S. 2010, MNRAS, 404, 1231

Walker, L. M., Johnson, K. E., Gallagher, S. C., et al. 2010, AJ, 140, 1254 